in vivo $31: 55-60(2017)$

doi:10.21873/invivo.11025

\title{
Wound Healing Delay in the ZDSD Rat
}

\author{
MARK A. SUCKOW ${ }^{1}$, TROY A. GOBBETT ${ }^{2}$ and RICHARD G. PETERSON ${ }^{2}$ \\ ${ }^{1}$ Department of Veterinary Population Medicine, University of Minnesota, St. Paul, MN, U.S.A.; \\ ${ }^{2}$ PreClinOmics, Inc., Indianapolis, IN, U.S.A.
}

\begin{abstract}
Animal models of diabetic delayed wound healing are essential to the development of strategies to improve clinical approaches for human patients. The Zucker diabetic Sprague Dawley (ZDSD) rat has proved to be an accurate model of dietinduced obesity and diabetes and we evaluated the utility of the $Z D S D$ rat as a model for delayed wound healing associated with diabetes and obesity. Groups of ZDSD and Sprague Dawley (SD) rats were placed on a diabetogenic diet and evaluated two weeks later for hyperglycemia, as a sign of diabetes. Rats with blood glucose levels of $>300 \mathrm{mg} / \mathrm{dl}$ were considered diabetic and those with blood glucose of $<180 \mathrm{mg} / \mathrm{dl}$ were considered nondiabetic. All SD rats were non-diabetic. A full-thickness excisional skin wound was created in anesthetized rats using a punch biopsy and wound diameter measured on days 1, 4, 7, 9 and 11. Blood glucose levels and body weights were measured periodically before and after wounding. Diabetic ZDSD rats had significantly greater blood glucose levels than non-diabetic $Z D S D$ and SD rats within 10 days of being placed on the diabetogenic diet. Furthermore, diabetic ZDSD rats initially weighed more than non-diabetic ZDSD and SD rats, however, by the end of the study there was no significant difference in body weight between the ZDSD groups. By day nine, wounds in $Z D S D$ rats were significantly larger than those in SD rats and this persisted until the end of the study at day fourteen. Wounds from all groups were characterized histologically by abundant fibroblast cells, collagen deposition and macrophages. These results demonstrate delayed wound healing in both diabetic and non-diabetic ZDSD rats and suggest that obesity or metabolic syndrome are important factors in wound healing delay.
\end{abstract}

It is estimated that there are more than 400 million or more people in the world suffering from diabetes mellitus (1). Many

This article is freely accessible online.

Correspondence to: Dr. Mark A. Suckow, Department of Veterinary Population Medicine, University of Minnesota, 1365 Gortner Avenue, St. Paul, MN 55108, U.S.A. E-mail: msuckowd@umn.edu

Key Words: Diabetes, obesity, wound healing, zucker diabetic sprague dawley, rat. diabetic patients have difficulty in healing wounds and the annual cost to manage these wounds exceeds 20 billion dollars (2). In particular, wounds affecting the feet are common, with amputation being a frequent outcome (3). Because of the importance of impaired wound healing in diabetics, animal models of diabetic wound healing are of great interest.

Rodent models of diabetic wound healing have received a great deal of focus due to ease of maintenance, cost and availability of genetically modified lines. The full-thickness excisional wound model is a standard approach in which a punch biopsy is used to remove both epidermal and dermal tissues, allowing evaluation of epithelialization, granulation, and angiogenesis, all key processes during physiological wound healing (4). Although wound healing in rodents occurs mainly by contraction, several diabetic rodent models have been used to characterize wound healing in type 2 diabetes, including the $d b / d b$ mouse, the JCR:LA-cp rat and the Zucker diabetic fatty (ZDF) rat (5-8).

The Zucker diabetic Sprague dawley (ZDSD) rat is an animal model of type 2 diabetes and dietary obesity which develop subsequently to chronic dietary manipulation $(9,10)$. ZDSD rats were developed by cross-breeding diet-induced obesity (DIO) rats derived from Sprague Dawley rats $\mathrm{Crl}: \mathrm{CD}(\mathrm{SD})$ with $\mathrm{ZDF}^{+/+}$lean rats. Lacking leptin receptor defects, the ZDSD rat possesses an obese phenotype and the potential to develop overt hyperglycemia between 15 and 21 weeks of age. These characteristics make the ZDSD rat an excellent model of human adult-onset diabetes which develops over time in response to chronic overeating and lack of physical activity. The studies described here were undertaken to determine if the ZDSD rat demonstrates impaired wound healing and might, therefore, be useful as an animal model of delayed healing in type- 2 diabetic patients.

\section{Materials and Methods}

Animals. Four month old male rats documented to be free of common infectious pathogens were used. All studies were approved by the Institutional Animal Care and Use Committee. Rats were maintained in solid-bottom polycarbonate cages containing abundant hardwood chip bedding. ZDSD rats were obtained from PreClinOmics, Inc., (now a Crown Bioscience company; Indianapolis, IN, USA) and Hsd:Sprague Dawley ${ }^{\circledR} \mathrm{SD}^{\circledR}$ rats were 
obtained from Harlan, Inc., (Indianapolis, IN, USA). They were allowed ad libitum access to fresh water. All animals (ZDSD and SD) were provided TestDiet 5SCA (PMI Nutrition, International, St. Louis, MO, USA) for two weeks when they were 17-19 weeks of age. Heavier ZDSD rats developed diabetes while lighter ZDSD rats remained non-diabetic and were used as a control group. After two weeks on the 5SCA diet, all animals were placed on LabDiet 5008 (PMI Nutrition) for the remainder of the study.

Animals were weighed and blood glucose levels were determined with a glucometer from tail vein samples, weekly. Rats with blood glucose levels exceeding $300 \mathrm{mg} / \mathrm{dl}$ were considered diabetic and those with levels below $180 \mathrm{mg} / \mathrm{dl}$ were considered non-diabetic. Animals with blood glucose levels between $180 \mathrm{mg} / \mathrm{dl}$ and $300 \mathrm{mg} / \mathrm{dl}$ were excluded from the study.

Glucose measurement. Serum from whole blood samples was analyzed for glucose levels using a hand-held glucometer (Alpha TRAK 2, Abbott, Animal Health, North Chicago, IL). A drop of blood for glucose measurement was obtained after removing the skin from the tip of the tail $(<1 \mathrm{~mm})$. Measurements were performed on days $-17,-10,-3,4$, and 11 .

Excisional wound model. To create wounds, rats were anesthetized with isoflurane administered via a precision vaporizer and prepared for aseptic surgery. Using a 6-mm punch biopsy instrument (Miltex, Inc., York, PA, USA), a full-thickness skin wound was created in the dorsal thorax of each animal. Rats were administered ketoprofen at 4 $\mathrm{mg} / \mathrm{kg}$ subcutaneously immediately following surgery and again 8 hours post-operatively. Wounds were left uncovered following surgery. Wound diameter was measured using a digital caliper on days $0,4,7,9,11$, and 14 following surgery, with the day of wounding considered to be day 0 . Animals were weighed prior to initiation of the study and again weekly over the course of the two-week study. On day 14 , rats were euthanized with an overdose of inhaled carbon dioxide and wound sites harvested and fixed in $10 \%$ neutral buffered formalin. Tissues were sectioned at 4-5 $\mu \mathrm{m}$, stained with hematoxylin and eosin and subjectively evaluated for histological character.

Statistical analysis. Wound diameter values were compared for differences between groups using the Wilcoxon rank sum test. Statistical significance was reached when $p \leq 0.05$. All are presented as mean \pm standard error of the mean (SEM).

\section{Results}

Body weight. Body weights over the course of the study are summarized in Figure 1. The ZDSD rats became obese when compared to the SD controls. At the initiation of the diabetogenic 5SCA diet, the ZDSD rats that became diabetic weighed significantly more (490.60 g $\pm 4.44 \mathrm{SEM} ; p \leq 0.05)$ than the ZDSD rats that did not become diabetic (415.17 $\mathrm{g}$ $\pm 11.06 \mathrm{SEM})$ and the SD rats $(383.70 \mathrm{~g} \pm 6.80 \mathrm{SEM})$. Furthermore, the ZDSD rats that did not become diabetic weighed significantly more $(p \leq 0.02)$ than the SD rats. This trend persisted during and after the time that animals were provided the 5SCA diet and the 5008 diet; however by the time of euthanasia, there was no significant difference between the weight of diabetic (464.72 $\mathrm{g} \pm 6.26)$ and non-

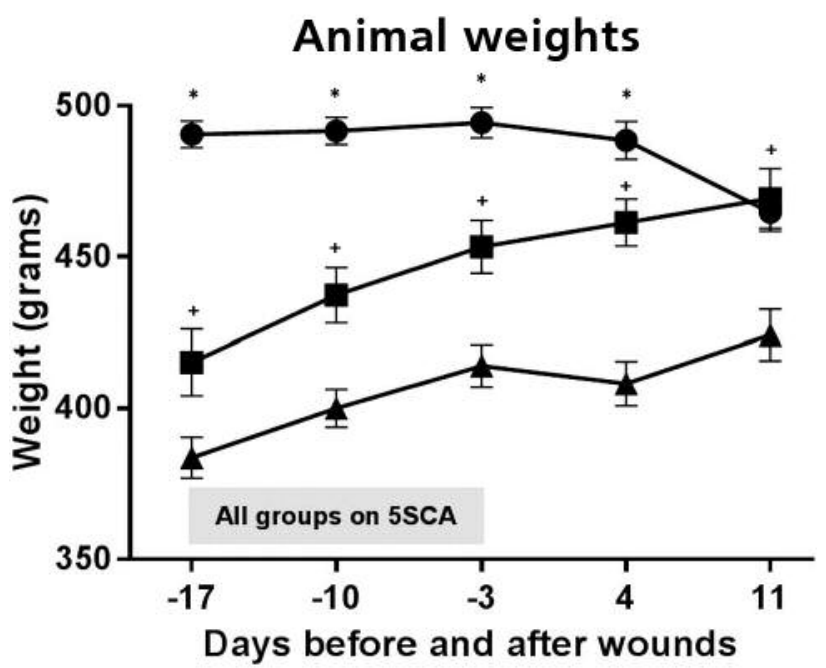

Figure 1. Body weights of diabetic ZDSD (-), non-diabetic ZDSD ( and $S D(\mathbf{\Delta})$ rats over the course of the study. The shaded bar indicated the period of time when all of the groups of animals were on the diabetogenic diet (5SCA). The more obese ZDSD rats became diabetic when placed on the diabetogenic diet versus the non-diabetic ZDSD and $S D$ controls. At the initiation of the diabetogenic 5SCA diet, the ZDSD rats that became diabetic weighted significantly more $(p \leq 0.05)$ than both the ZDSD rats that did not become diabetic and the SD rats. The $Z D S D$ rats however, that did not become diabetic weighed significantly more $(p \leq 0.02)$ than the SD rats. This trend persisted during and after the time that animals were provided the 5SCA diet and the 5008 diet; however, by the time of euthanasia, there was no significant difference between the weight of diabetic and non-diabetic ZDSD rats. Both groups of $Z D S D$ rats weighed significantly more $(p \leq 0.02)$ than $S D$ rats at the time of euthanasia. The * symbol indicates significantly greater body weight compared to non-diabetic ZDSD and SD rats; and the + symbol indicates significantly greater body weight compared to SD rats.

diabetic (469.31 g \pm 9.91$)$ ZDSD rats. Both groups of ZDSD rats weighed significantly more $(p \leq 0.02)$ than SD rats $(424.24 \mathrm{~g} \pm 8.47)$ at the time of euthanasia.

Blood glucose. Rats with blood glucose exceeding $300 \mathrm{mg} / \mathrm{dl}$ were considered diabetic and those with levels below $180 \mathrm{mg} / \mathrm{dl}$ were considered non-diabetic. As shown in Figure 2 , all animals had similar blood glucose values prior to being placed on the 5SCA diet; however, within 7 days blood glucose values were significantly greater $(p \leq 0.001)$ in diabetic ZDSD rats $(392.03 \mathrm{mg} / \mathrm{dl} \pm 11.72 \mathrm{SEM})$ than in non-diabetic ZDSD rats $(148.77 \mathrm{mg} / \mathrm{dl} \pm 2.80 \mathrm{SEM})$ and SD rats $(120.34$ $\mathrm{mg} / \mathrm{dl} \pm 2.06 \mathrm{SEM})$. This trend persisted through the remainder of the study. Values were not significantly different between non-diabetic ZDSD rats and SD rats at any time point.

Wound diameter. To determine if wound closure was delayed in diabetic ZDSD rats, 6-mm punch biopsy wounds were created and wound diameter measured periodically through 


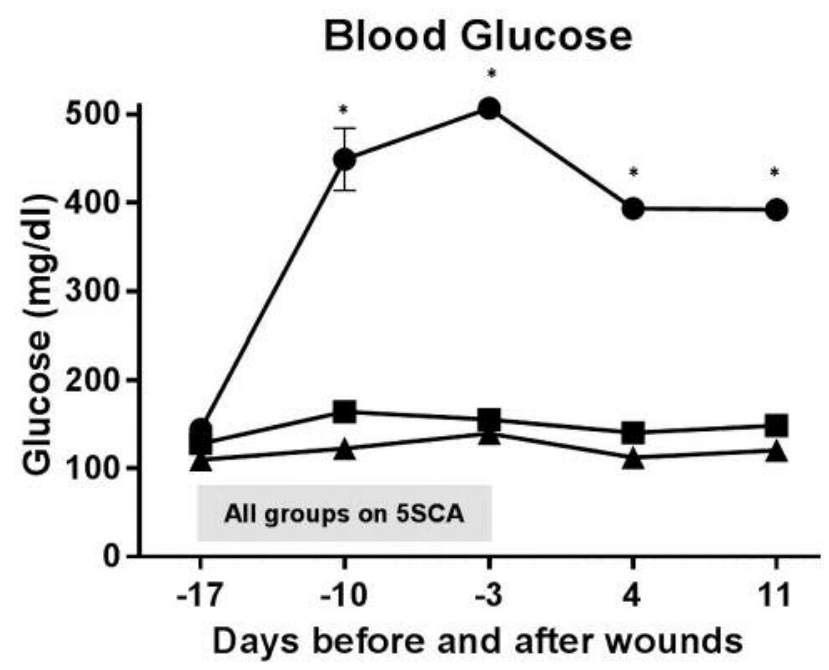

Figure 2. Glucose levels of all the groups of animals during the whole experiment. The shaded bar indicated the period of time when all of the groups of animals were on the diabetogenic diet (5SCA). The diabetic ZDSD (-) rats had consistently higher glucose levels than the nondiabetic ZDSD (ם) and SD (ט) rats. All animals had similar blood glucose values prior to being placed on the 5SCA diet; however, within 7 days, blood glucose values were significantly greater $(p \leq 0.001)$ in diabetic $Z D S D$ rats than in non-diabetic ZDSD rats and SD rats. This trend persisted through the remainder of the study. Values were not statistically different between non-diabetic ZDSD rats and SD rats at any experimental time point. The * symbol indicates significantly greater blood glucose levels in ZDSD rats compared to non-diabetic ZDSD and SD rats.

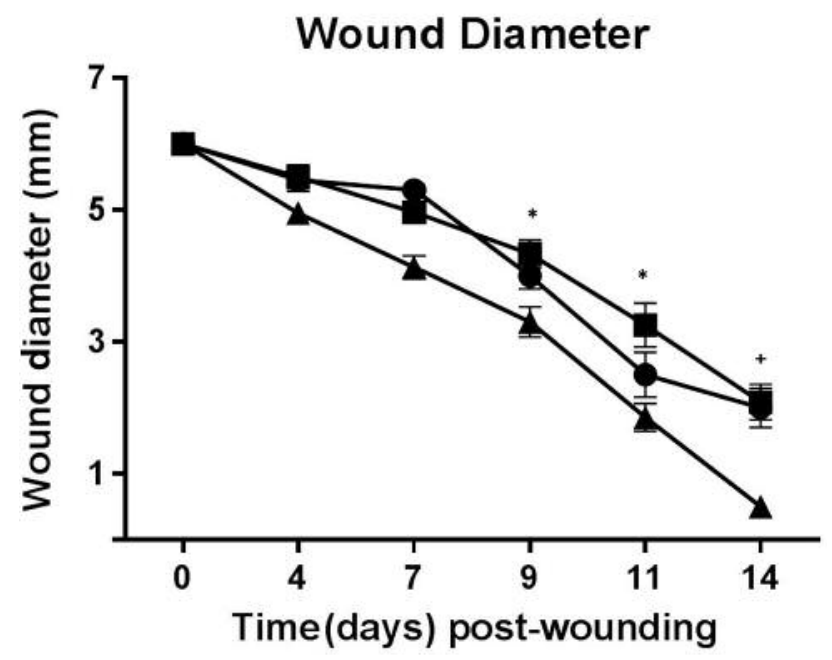

Figure 3. Wound healing analysis over the course of the study. There were no significant differences in mean wound diameter between any groups until day 7 after injury; however, diabetic $(\bullet)$ and non-diabetic ZDSD (ם) rats had significantly $(p \leq 0.05)$ larger wounds than $S D(\mathbf{\Delta})$ rats on days 9 and 11 , as well as on day $14(p<0.01)$. Wound size between diabetic and nondiabetic ZDSD rats did not differ significantly at any experimental time point. The * symbol indicates significant difference of $p \leq 0.05$, and the + symbol indicates significant difference of $p \leq 0.01$.

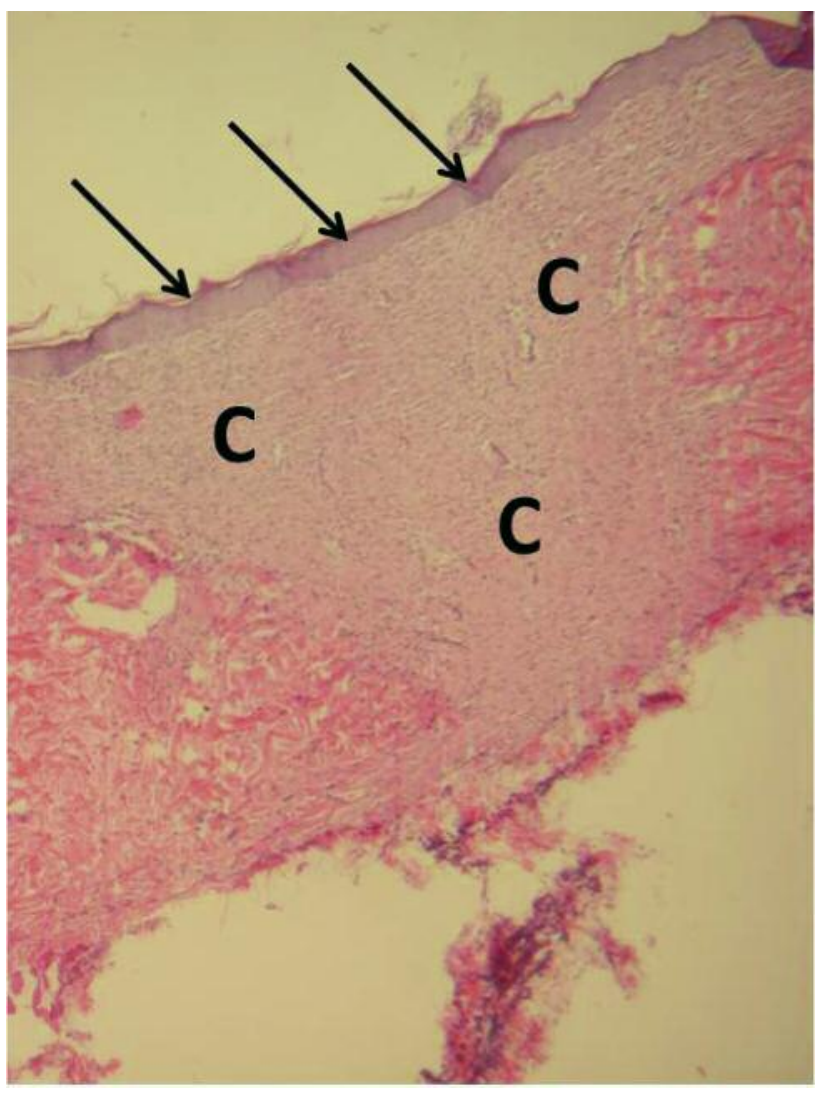

Figure 4. Photomicrograph of healed wound site from a diabetic ZDSD rat demonstrating complete re-epithelialization (arrows) overlying abundant connective tissue $(C)(100 \times$, stained with hematoxylin and eosin).

14 days. By day 14 , most wounds still had at least small incompletely healed wound areas remaining, although wounds in 4/10 rats in the SD group had completely healed. No clinical evidence of infection was present in any of the wounds at any timepoint. As shown in Figure 3, wound diameter progressively decreased in all groups of rats through the study period. There were no significant differences in mean wound diameter between any groups through day 7 after injury; however, diabetic and nondiabetic ZDSD rats had significantly larger $(p \leq 0.05)$ incompletely healed wounds than SD rats on days 9 and 11, as well as on day $14(p \leq 0.01)$. Wound size between diabetic and non-diabetic ZDSD rats did not differ significantly at any time point.

Wound histology. Wound tissues were sampled on day 14 at the time of euthanasia, processed, and examined for histological change. Wounds from all groups demonstrated a normal healing process (Figure 4) characterized by re-epithelialization, abundant fibroblasts, collagen, and occasional polymorphonuclear cells, macrophages, and giant cells. 


\section{Discussion}

Diabetic patients suffer a number of complications including renal disease (11), cardiovascular disease (12), and impaired wound healing, particularly foot ulcers (13, 14). Multiple factors exist that relate to impaired wound healing, including abnormal glucose metabolism and vascular complications. For example, hyperglycemia initiates events that result in impaired collagen synthesis (15); and impaired vascular function in diabetics is associated with thickening of the basement membrane of the capillaries and arterioles (16).

Diabetes in animal models occurs as either the result of experimental manipulation or inherited genetic trait. For example, administration of streptozotocin has been shown to reliably produce diabetes in mice, rats, and pigs (17-20). In contrast, the non-obese diabetic (NOD) and the leptindeficient $(o b / o b)$ lines are examples of commonly used animal models with inherited propensity to development of diabetes mellitus (22-24).

Animal models have been described for healing of excisional (25-27), incisional $(28,29)$, and burn wounds $(30,31)$, and a major emphasis has been placed on healing in diabetic subjects. Excisional wound models are the most common, largely because wound creation is straightforward and processes such as epithelialization, angiogenesis, and scar formation can be readily assessed (32). Though excisional wounds in humans heal primarily by epithelialization, contraction of the wound edges through the action of myofibroblasts is an important facet of wound healing in rodents. Nonetheless, rodents are widely used for wound healing studies because of expense, ease of maintenance, availability of inbred and genetically modified lines, and standardized methods for induction of diabetes and related complications (33). The $d b / d b$ mouse, a monogenic animal model of type 2 diabetes, with obesity and early insulin resistance, has been the primary model used for studies of impaired wound healing associated with diabetes (34-37). Because of planned studies designed to evaluate a hernia repair material in a diabetic model, we required an animal larger than a mouse. We first needed to confirm impaired wound healing ability in this model, and therefore conducted the present study to quantitatively document that phenotype.

The ZDSD rat is characterized by rapid weight gain on standard rodent chow, insulin resistance before hyperglycemia develops, and hypoinsulinemia as diabetes develops (10). Further, the ZDSD rat lacks leptin receptor defects typical of some other models of diabetes. We found that most ZDSD rats became hyperglycemic by 17 weeks of age, though a small number $(\mathrm{N}=6)$ were not and, therefore, considered to be non-diabetic. Both diabetic and non-diabetic ZDSD rats became obese, thus supporting the idea that, in this model, diabetes and obesity are inherited separately. Diabetic and non-diabetic ZDSD rats weighed significantly more than SD rats by day 7 of the study, but there was no difference in body weight between either type of ZDSD rat.

Following creation of full-thickness dermal wounds, healing was evaluated by measurement of wound diameter. Wounds in SD rats closed quickly, with 4/10 wounds completely healed by 14 days after wounding. In contrast, wounds in diabetic and non-diabetic ZDSD rats healed more slowly than those in SD rats; and there was no difference in wound healing rates between either type of ZDSD rat, thus suggesting that impaired wound healing was not dependent on presence of diabetes. Histological change was characterized by fibroplasia with a mixed inflammatory response; and there was no difference between wound sites of rats in any of the groups.

Others have demonstrated impaired excisional wound healing in obese diabetic versus lean non-diabetic ZDF rats, and it was suggested that the presence of a large amount of adipose in wounds of diabetic rats may have contributed a different fibroblast phenotype in diabetic wounds resulting in impaired wound healing (8). Our results support that conclusion, as both diabetic and non-diabetic ZDSD rats were obese and had similar degrees of impaired wound healing. Thus, in the ZDSD rat, obesity appears to be a determining factor in delayed wound healing. This is consistent with results showing that obesity in non-diabetic Wistar rats delayed wound healing compared to non-obese controls (38). The role of obesity in delayed wound healing in obese rats appears to be related to the association of abundant adipose tissue with chronic low-grade inflammation $(39,40)$. Increased levels of mediators of inflammation, such as TNF- $\alpha$ and IL-6, occur in obese humans and may account for this effect $(41,42)$. The precise mechanism of delayed wound healing related to obesity the ZDSD rat is unclear, and further studies are needed to define that process.

\section{Acknowledgements}

The Authors wish to acknowledge the technical assistance of Kathryn Coy for completion of these studies.

\section{References}

1 NCD Risk Factor Collaboration: Worldwide trends in diabetes since 1980: a pooled analysis of 751 populationbased studies with 4.4 million participants. Lancet 387 : 1513-1530, 2016.

2 Harding KG, Morris HL and Patel GK: Science, medicine, and future: healing chronic wounds. BMJ 324: 160-163, 2002.

3 Game F: Choosing life or limb. Improving survival in the multicomplex diabetic foot patient. Diabetes Metab Res Rev 28(Suppl 1): 97-100, 2012. 
4 Wong VW, Sorkin M, Glotzbach JP, Longaker MT and Gurtner GC: Surgical approaches to create murine models of human wound healing. J Biomed Biotechnol 2011: 969618, 2011.

5 Bauer BS, Ghahary A, Scott PG, Iwashina T, Demare J, Russell JC and Tredget EE: The JCR:LA-cp rat: A novel model for impaired wound healing. Wound Rep Reg 12: 86-92, 2004.

6 Fang RC, Kryger ZB, Buck DW, De La Garza M, Galiano RD and Mustoe TA: Limitations of the $d b / d b$ mouse in translational wound healing research: is the NONcNZO10 polygenic mouse model superior? Wound Rep Reg 18: 605-613, 2010.

7 Marrotte EJ, Chen D-D, Hakim JS and Chen AF: Manganese superoxide dismutase expression in endothelial progenitor cells accelerates wound healing in diabetic mice. J Clin Invest 120 : 4207-4219, 2010.

8 Slavkovsky R, Kohlerova R, Tkacova V, Jiroutova A, Tahmazoglu T, Velebny V, Rezacova M and Sobotka L: Zucker diabetic fatty rat: A new model of impaired cutaneous wound repair with type II diabetes mellitus and obesity. Wound Rep Reg 19: 515-525, 2011.

9 Reinwald S, Peterson RG, Allen MR and Burr DB: Skeletal changes associated with the onset of type 2 diabetes in the ZDF and ZDSD rodent models. Am J Physiol Endocrinol Metab 296: E765-E774, 2009.

10 Peterson RG, Jackson CV, Zimmerman K, de Winter W, Huebert $\mathrm{N}$ and Hansen MK: Characterization of the ZDSD rat: $\mathrm{A}$ translational model for the study of metabolic syndrome and type 2 diabetes. J Diabetes Res 2015: 487816, 2015.

11 Alpers CE and Hudkins KL: Mouse models of diabetic nephropathy. Curr Opin Nephrol Hypertens 20: 278-284, 2011.

12 Kannel WB and McGee DL: Diabetes and cardiovascular risk factors: the Framingham study. Circulation 59: 8-13, 1979.

13 Cavanagh PR, Lipsky BA, Bradbury AW and Botek G: Treatment for diabetic foot ulcers. Lancet 366: 1725-1735, 2005.

14 Singh N, Armstrong DG and Lipsky BA: Preventing foot ulcers in patients with diabetes. JAMA 293: 217-228, 2005

15 Hennessey PJ, Ford EG, Black CT, and Andrassy RJ: Wound collagenase activity correlates directly with collagen glycosylation in diabetic rats. J Ped Surg 25: 75-78, 1990.

16 Flynn MD and Tooke JE: Aetiology of diabetic foot ulceration: a role for the microcirculation? Diabetic Med 9: 320-329, 1992.

17 Jakobsen $\mathrm{J}$ and Lundbeck K: Neuropathy in experimental diabetes: an animal model. Br Med J 2: 278-279, 1976.

18 Wei M, Smith MT, Ross FB, Schmid K, Hoey AJ, Burstow D and Brown L: The streptozotocin-diabetic rat as a model of the chronic complications of human diabetes. Heart Lung Circ 12: 44-50, 2003.

19 Vereniuk I, Pavlov IA and Obrosova IG: Inducible nitric oxide synthase gene deficiency counteracts multiple manifestations of peripheral neuropathy in a streptozotocin-induced mouse model of diabetes. Diabetologia 51: 2126-2133, 2008.

20 von Wilmowsky C, Stockman P, Metzler P, Harsch I A, Armann $\mathrm{K}$ and Schlegel KA: Establishment of a streptozotocin-induced diabetic domestic pig model and a systematic evaluation of pathological changes in the hard and soft tissue over a 12-month period. Clin Oral Implants Res 21: 709-717, 2010.

21 Lee MS, Song KD, Yang HJ, Solis CD, Kim SH and Lee WK: Development of a type II diabetic mellitus animal model using Micropig ${ }^{\circledR}$. Lab Anim Res 28: 205-208, 2012.

22 Schmidt RE, Dorsey DA, Beaudet LN, Frederick KE, Parvin CA, Plurad SB and Levisetti MG: Non-obese diabetic mice rapidly develop dramatic sympathetic neuritic dystrophy: a new experimental model of diabetic autonomic neuropathy. Am J Pathol 163: 2077-2091, 2003.

23 Drel VR, Mashtalir N, Ilnytska O, Shin J, Li F, Lyzogubov VV and Obrosova IG: The leptin-deficient $(o b / o b)$ mouse: a new animal model of peripheral neuropathy of type 2 diabetes and obesity. Diabetes 55: 3335-3343, 2006.

24 Homs J, Arize L, Pages G, Verdú E, Casals L, Udina E, Chillón M, Bosch A and Navarro X: Comparative study of peripheral neuropathy and nerve regeneration in NOD and ICR diabetic mice. J Peripher Nerv Syst 16: 213-227, 2011.

25 Cross SE, Naylor IL, Coleman RA and Teo TC: An experimental model to investigate the dynamics of wound contraction. Br J Plast Surg 48: 184-197, 1995.

26 Stiernberg J, Norfleet AM, Redin WR, Warner WS, Fritz RR and Carney DH: Acceleration of full-thickness wound healing in normal rats by the synthetic thrombin peptide, TP508. Wound Rep Reg 8: 204-215, 2000.

27 Kirchner LM, Meerbaum SO, Gruber BS, Knoll AK, Bulgrin J, Taylor RA and Schmidt SP: Effects of vascular endothelial growth factor on wound closure rates in the genetically diabetic mouse model. Wound Rep Reg 11: 127-131, 2003.

28 Quaglino D Jr., Nanney LB, Ditesheim JA and Davidson JM: Transforming growth factor-beta stimulates wound healing and modulates extracellular matrix gene expression in pig skin: incisional wound model. J Invest Dermatol 97: 34-42. 1991.

29 Gottrup F, Agren MS and Karlsmark T: Models for use in wound healing research: a survey focusing on in vitro and in vivo adult soft tissue. Wound Rep Reg 8: 83-96, 2000.

30 Stieritz DD and Holder IA: Experimental studies on the pathogenesis of infections due to Pseudomonas aeruginosa: description of a burned mouse model. J Infect Dis 131: 688-691, 1975.

31 Oluwasanmi $\mathrm{J}$ and Chvapil M: A comparative study of four materials in local burn care in rabbit model. J Trauma 16: 348353, 1976.

32 Greenhalgh DG and Warden GD: Wound care models; in Surgical research (eds) W W Souba and D W Wilmore (London: Academic Press) pp. 379-391, 2001.

33 Hsueh W, Abel ED, Breslow JL, Maeda N, Davis RC, Fisher EA, Dansky H, McClain DA, McIndoe R, Wassef MK, Rabadán-Diehl C, and Goldberg IJ: Recipes for creating animal models of diabetic cardiovascular disease. Circ Res 100: 14151427, 2007.

34 Coleman DL: Diabetes-obesity syndromes in mice. Diabetes 31: $1-6,1982$

35 Galliano RD, Michaels J, Dobryansky M, Levine J P and Gurtner GC: Quantitative and reproducible murine model of excisional wound healing. Wound Rep Reg 12: 485-492, 2004.

36 Carley AN and Severson DL: Fatty acid metabolism is enhanced in type 2 diabetic hearts. Biochim Biophys Acta 1734: 112-126, 2005.

37 Michaels J, Churgin SS, Blechman KM, Greives MR, Aarabi S, Galiano RD and Gurtner GC: $d b / d b$ mice exhibit severe woundhealing impairments compared with other murine diabetic strains in a silicone-splinted excisional wound model. Wound Rep Reg 15: 665-670, 2007.

38 Nascimento AP and Costa AM: Overweight induced by high-fat diet delays rat cutaneous wound healing. Br J Nutr 96: 10691077, 2006. 
39 Dandona P, Weinstock R, Thusu K, Abdel-Rahman E, Aljada A and Wadden T: Tumor necrosis factor-alpha in sera of obese patients: fall with weight loss. J Clin Endocrinol Metab 83: 2907-2910, 1998.

40 Yudkin JS, Kumari M, Humphries SE and Mohamed-Ali V: Inflammation, obesity, stress and coronary heart disease: is interleukin-6 the link? Atherosclerosis 148: 209-214, 2000.

41 Das UN: Obesity, metabolic syndrome X, and inflammation. Nutrition 18: 430-432, 2002.
42 Liu J, Divoux A, Sun J, Zhang J, Clément K, Glickman JN, Sukhova GK, Wolters PJ, Du J, Gorgun CZ, Doria A, Libby P, Blumberg RS, Kahn BB, Hotamisligil GS and Shi GP: Genetic deficiency and pharmacological stabilization of mast cells reduce diet induced obesity and diabetes in mice. Nat Med 15: 940-945, 2009.

Received October 28, 2016

Revised November 14, 2016

Accepted December 6, 2016 